\title{
Perfil motivacional e estado de humor em corredores de rua integrantes de grupos de corrida
}

\author{
Motivational profile and mood in running group participants
}

\author{
Jolnes Neumann Gula ${ }^{1}$ \\ Verônica Volski Mattes ${ }^{2}$ \\ Ana Carolina Paludo ${ }^{3}$ \\ Michael Pereira da Silva ${ }^{4}$ \\ Marcus Peikriszwili Tartaruga ${ }^{5}$ \\ Endereço para correspondência \\ Jolnes Neumann Gula \\ Rua Simeão Camargo Varela de Sá, 03 - Bairro Vila Carli \\ 85040-080 - Guarapuava, PR [Brasil]. +55 42 99809-6175 \\ jolnesneumann@gmail.com
}

Graduado em Educação Física Bacharelado, Grupo de Pesquisa em Biomecânica e Energética do Movimento Humano, Curso de Educação Física Bacharelado - Universidade Estadual do CentroOeste - UNICENTRO. Guarapuava, PR - Brasil.

jolnesneumann@gmail.com

Mestre em Educação, Grupo de Pesquisa em Biomecânica e Energética do Movimento Humano, Curso de Educação Física Bacharelado - Universidade Estadual do Centro-Oeste UNICENTRO. Guarapuava, PR - Brasil.

vvolski@unicentro.br

Doutora em Educação Física, Grupo de Pesquisa em Biomecânica e Energética do Movimento Humano, Curso de Educação Física Bacharelado - Universidade Estadual do CentroOeste - UNICENTRO. Guarapuava, PR - Brasil. anacpaludo@gmail.com

Doutor em Educação Física, Grupo de Pesquisa em Biomecânica e Energética do Movimento Humano, Faculdade de Medicina - FAMed - Universidade Federal de Rio Grande - FURG. Rio Grande, RS - Brasil. prof_mpsilva@outlook.com

Doutor em Educação Física, Grupo de Pesquisa em Biomecânica e Energética do Movimento Humano, Curso de Educação Física Bacharelado - Universidade Estadual do CentroOeste - UNICENTRO. Guarapuava, PR - Brasil.

mtartaruga@unicentro.br

\section{Resumo}

Introdução: A busca pela prática corrida de rua vem aumentando e um melhor entendimento do perfil dos praticantes pode auxiliar na melhor adesão dos participantes à prática da atividade física.

Objetivo: investigar o perfil motivacional e estado de humor de corredores de rua.

Materiais e Métodos: foram avaliados 50 participantes de uma prova de corrida de rua. A motivação e o estado de humor foram avaliados após a prova pelos questionários IMPRAF54 e POMS, respectivamente. As dimensões da motivação foi avaliada pelo teste de Wilcoxon e o humor foi analisado de maneira descritivas.

Resultados: as dimensões mais presentes na motivação foram a Saúde e o Prazer. Para o humor foi observado um perfil iceberg.

Conclusão: Com base nos resultados, os corredores de rua do presente estudo apresentam motivação a prática da corrida de rua pela busca da Saúde e Prazer, adicionalmente nota-se um estado de humor positivo dos praticantes.

Descritores: Motivação. Corrida. Psicologia do Esporte.

\section{Abstract}

Introduction: Increasing attention is being paid to street running race practice, and a better understanding of the practitioner profile can help participants adhere to the practice of physical activity.

Objective: To investigate the motivational profile and mood of street runners.

Material and Methods: 50 participants in a street race event were evaluated. Motivation and mood were assessed after the race using the IMPRAF-54 and POMS questionnaires, respectively. The dimensions of motivation were assessed using the Wilcoxon test, and mood was analyzed in a descriptive approach.

Results: The dimensions most present in motivation were health and pleasure. For mood, an iceberg profile was observed.

Conclusion: Based on the results, the street runners in this study presented a motivation of the pursuit of health and pleasure in the practice of street running; in addition, the practitioners showed a positive mood.

Keywords: Motivation. Running. Psychology sport.

Cite como

Vancouver

Gula JN, Mattes VV, Paludo AC, Silva MP, Tartaruga MP. Perfil motivacional e estado de humor em corredores de rua integrantes de grupos de corrida. Conscientiae Saúde 2019 out./dez.;18(4):444-454. https://doi.org/10.5585/conssaude.v18n4.14826. 


\section{Introdução}

Segundo as Diretrizes do Colégio Americano de Medicina do Esporte (ACSM), a prática de atividade física e/ou exercício regularmente resulta em múltiplos benefícios para a saúde ${ }^{1}$, além de ser um fator que contribui para a qualidade de vida dos indivíduos ${ }^{2}$. Em meio a diversas modalidades de exercício físico, a prática de corrida vem apresentando grande crescimento, principalmente entre a população adulta ${ }^{3}$. $\mathrm{O}$ aumento do número de praticantes de corrida pode estar atrelado às próprias características do exercício, como seu baixo custo e disponibilidade para a prática, assim como por seus benefícios fisiológicos e psicológicos ${ }^{4,5}$. Dentre as formas de corrida, destaca-se a corrida de rua, prova de pedestrianismo disputada em circuitos de ruas, avenidas e estradas, com distâncias oficiais que podem variar entre 5 a $100 \mathrm{Km}^{6,7}$.

Compreender os motivos que levam os indivíduos à busca pela prática da corrida de rua se torna importante para que os mesmos possam usufruir melhor e por mais tempo dos benefícios da prática desta modalidade ${ }^{8,9}$. Diversos estudos buscaram avaliar a motivação de atletas maratonistas ${ }^{10,11}$ e ultramaratonistas ${ }^{12,13}$, porém, ainda são poucos os estudos que investigaram a motivação de corredores recreacionais de rua ${ }^{14,15}$ ou corredores vinculados à grupos de corrida ${ }^{8}$.

Entende-se como motivação o conjunto de considerações que levam a pessoa a realizar uma tarefa, sendo um fator cognitivo crítico na adoção e manutenção de uma rotina de exercícios ${ }^{16}$. Balbinotti ${ }^{17}$ sugere que as dimensões motivacionais relacionadas ao entendimento da prática regular de atividades físicas ou esportivas são: controle de estresse, saúde, sociabilidade, competitividade, estética e prazer. Seus pressupostos são fundamentados pela Teoria da Auto Determinação (Self-Determination Theory), descrita por Deci \& Ryan ${ }^{18,19}$, que distingue diferentes tipos de motivação. Segundo essa teoria, os indivíduos podem ser motivados em diferentes níveis, sendo eles: intrinsecamente motivados (quando o sujeito ingressa na atividade pela sua própria vontade, pelo prazer e pelo processo de conhecer uma nova atividade); extrinsecamente motivados (quando o sujeito realiza a atividade com outro objetivo que não o inerente à própria pessoa); ou, então, ser amotivados (quando o indivíduo não identifica nenhum bom motivo para realizar a atividade) ${ }^{18,19}$.

Uma maior motivação para a prática de um exercício físico ou esporte pode resultar num comportamento mais ativo. A adoção e manutenção de um comportamento ativo pode levar a uma maior percepção de prazer e satisfação por parte do praticante, o que pode consequentemente gerar uma resposta positiva no domínio psicológico. Dentre as variáveis psicológicas influenciadas positivamente pela prática do exercício físico destaca-se o estado de 
humor. Alguns estudos têm demonstrado que maiores níveis de atividade física estão relacionados a um estado humor positivo quando comparados a indivíduos menos ativos fisicamente ${ }^{20}$, entretanto, isso não é um consenso na literatura ${ }^{21-23}$. Adicionalmente, ainda existe uma lacuna na literatura, no que diz respeito às respostas de estado de humor em corredores recreacionais de corrida de rua.

Assim, tendo em vista que a corrida de rua é uma das modalidades com crescimento significativo dentre a prática de atividades físicas em adultos, e que a motivação é um fator importante para a adesão à prática da modalidade, assim como a sua prática pode auxiliar na dimensão positiva do estado de humor, o presente estudo buscou avaliar o perfil motivacional e de estado de humor de corredores de rua inseridos em grupos de corrida.

\section{Material e Métodos}

\section{Participantes}

A amostra foi selecionada por conveniência, sendo composta por 50 corredores de rua de ambos os sexos (masculino $=23$; feminino $=27$ ) com idades variando entre 19 e 64 anos (média $=39,76 ; \mathrm{DP}=11,02$ ), participantes de diversos grupos de corrida de rua e assessorias esportivas da cidade de Guarapuava - Paraná. Como critério de inclusão para participação do presente estudo, considerou-se a inserção dos indivíduos em grupos de corrida de rua, bem como a apresentação de idade maior que 18 anos, além da assinatura do Termo de Consentimento Livre e Esclarecido (TCLE); e, como critérios de exclusão, considerou-se o preenchimento incompleto dos questionários aplicados. O estudo contou com a aprovação do Comitê de Ética em Pesquisa com Seres Humanos, sob parecer número 3.089.484 COMEP/UNICENTRO.

\section{Desenho do estudo}

O estudo caracterizou-se como transversal. Os treinadores e líderes dos grupos de corrida foram contatados previamente com o intuito de levantar o número de praticantes da modalidade bem como a disponibilidade para participar da coleta. As coletas ocorreram em apenas um momento, após uma prova beneficente de corrida de rua com percurso de cinco quilômetros, realizada no período da manhã. Os participantes foram abordados ao final da prova, em suas respectivas tendas de identificação de grupos de corrida de rua e assessorias esportivas. Após a explicação do objetivo do estudo, os participantes primeiramente 
preencheram e assinaram o TCLE e em seguida, os questionários de motivação (IMPRAF-54) e estado de humor (POMS).

\section{Instrumentos da pesquisa}

\section{Questionário biossociodemográfico}

O questionário socioeconômico foi aplicado com a finalidade de caracterização da amostra e controle das variáveis dependentes: "sexo", “idade”, "renda mensal”, “"escolaridade" e "tempo de prática”.

\section{Motivação para prática de atividade física}

Para avaliação da motivação para a prática da modalidade de corrida, utilizou-se o Inventário de Motivação à Prática Regular de Atividades Físicas (IMPRAF-54) proposto por Barbosa $^{24}$. O IMPRAF-54 é um questionário composto por 54 itens divididos em nove blocos, e subdivididos em seis domínios: controle de estresse, saúde, sociabilidade, competitividade, estética e prazer. Cada item é avaliado por meio de uma escala tipo Likert, bidirecional graduada em 5 pontos, indo de "isto me motiva pouquíssimo" (1) a "isto me motiva muitíssimo" (5). A validade e fidedignidade desse inventário foram testadas e demonstradas em um estudo prévio, apresentando valores aceitáveis ${ }^{24}$.

\section{Perfil de Humor}

O questionário de perfil do estado de humor POMS (Profile of Mood State) foi utilizado para descrição do perfil de humor dos participantes. O POMS é um instrumento amplamente utilizado na pesquisa com atividade física para medir as subescalas de humor ${ }^{25}$. Cada adjetivo é avaliado em uma escala de 5 pontos, indo de "Nunca" (0) a "Muitíssimo" (4). A versão do POMS utilizada neste trabalho corresponde a uma versão reduzida da escala original, sendo esta composta por 36 itens que avaliam seis escalas de humor: tensão, depressão, hostilidade, vigor, fadiga e confusão, além de 6 itens adicionais que compõem a escala de desajuste ao treino, perfazendo um total de 42 itens no questionário ${ }^{26}$.

\section{Análise estatística}

Para análise estatística utilizou-se o teste de Shapiro Wilk com o intuito de verificar a normalidade dos dados $(\mathrm{p}>0,05)$. Em seguida foram realizadas as análises descritivas, com 
mediana, mínimo e máximo, seguidas do teste de Wilcoxon signed-rank test para as comparações pareadas entre as dimensões. O nível de significância adotado foi de $p<0,05$. Para a análise dos dados de humor foram realizadas análises descritivas, com mediana, mínimo e máximo. Como a maioria das dimensões do questionário POMS não apresentou dados normais para a comparação entre os grupos, assumiu-se a estatística não paramétrica pelo teste de MannWhitney. Os dados foram analisados no pacote estatístico JASP, versão 0.8.2.

\section{Resultados}

Os participantes do estudo compreenderam idade entre 19 e 64 anos, estando a maioria com idades entre 35 e 50 anos. Adicionalmente, a amostra apresentou as seguintes características: a maioria dos participantes eram casados; com nível superior e pós-graduação; renda entre um e três salários mínimos; praticantes da modalidade de corrida de rua há mais de um ano; e inseridos em grupos de corrida há mais de um ano.

A Tabela 1 apresenta os resultados acerca das dimensões motivacionais dos participantes a partir das análises descritivas, com mediana, mínimo e máximo. Podemos observar que as dimensões de motivação para a prática de corrida que apresentaram maiores escores foram a Saúde e o Prazer para os homens, e a Saúde, o Prazer e a Sociabilidade para as mulheres.

Tabela 1 - Valores de mediana (mínimos e máximos) em escores das dimensões motivacionais dos participantes

\begin{tabular}{ccccccc}
\hline $\begin{array}{c}\text { Dimensões } \\
\text { Motivação }\end{array}$ & $\begin{array}{c}\text { Controle de } \\
\text { Estresse }\end{array}$ & Saúde & Sociabilidade & Competitividade & Estética & Prazer \\
\hline $\begin{array}{c}\text { Mediana } \\
\text { (mín-Máx) }\end{array}$ & $\begin{array}{c}\text { Mediana } \\
\text { (mín-Máx) }\end{array}$ & $\begin{array}{c}\text { Mediana } \\
\text { (mín-Máx) }\end{array}$ & $\begin{array}{c}\text { Mediana } \\
\text { (mín-Máx) }\end{array}$ & $\begin{array}{c}\text { Mediana } \\
\text { (mín-Máx) }\end{array}$ & $\begin{array}{c}\text { Mediana } \\
\text { (mín-Máx) }\end{array}$ \\
\cline { 2 - 7 } Geral & 32,0 & 37,0 & 33,0 & 17,5 & 29,5 & 35,0 \\
$(\mathrm{n}=50)$ & $(9,0-40,0)$ & $(23,0-40,0)$ & $(12,0-40,0)$ & $(8,0-38,0)$ & $(9,0-40,0)$ & $(23,0-40,0)$ \\
Homens & 31,0 & 36,0 & 29,0 & 20,0 & 28,0 & 33,0 \\
$(\mathrm{n}=23)$ & $(18,0-40,0)$ & $(28,0-40,0)$ & $(16,0-40,0)$ & $(8,0-37,0)$ & $(15,0-39,0)$ & $(25,0-40,0)$ \\
Mulheres & 32,0 & 38,0 & 36,0 & 17,0 & 31,0 & 36,0 \\
$(\mathrm{n}=27)$ & $(9,0-40,0)$ & $(23,0-40,0)$ & $(12,0-40,0)$ & $(8,0-38,0)$ & $(9,0-40,0)$ & $(23,0-40,0)$ \\
\hline
\end{tabular}

Fonte: Os autores, 2019.

Os resultados das comparações múltiplas realizadas por sexo entre as dimensões motivacionais são apresentados na Tabela 2. Nas comparações múltiplas realizadas para o sexo masculino, entre as dimensões de motivação para a prática de corrida, verificou-se que a Saúde foi a dimensão com maiores escores, sendo superior ao Controle de estresse, a Sociabilidade, a Competitividade e Estética. Adicionalmente, a segunda dimensão que mais motiva os corredores de rua é o Prazer, apresentando escores maiores em comparação com o Controle de 
Estresse, a Sociabilidade, e a Competitividade. A dimensão motivacional Controle de Estresse também apresentou diferenças significativas com maiores escores que a Competitividade e a Estética. As dimensões Sociabilidade e Estética apresentaram maiores escores com diferenças significativas apenas quando comparados à Competitividade.

Para o sexo feminino, as comparações múltiplas realizadas entre as dimensões de motivação mostraram que a Saúde foi a dimensão com maiores escores, sendo superior ao Controle de Estresse, a Competitividade e Estética. Observamos que dimensões Prazer e Sociabilidade apresentaram diferenças significativas, também com maiores escores que as dimensões Controle de Estresse, a Competitividade e Estética. As dimensões Controle de Estresse e Estética apresentaram diferenças significativas apenas quando comparadas a Competitividade.

Tabela 2 - Comparações múltiplas de escores de medianas por sexo entre as dimensões motivacionais

\begin{tabular}{|c|c|c|c|c|c|c|c|c|c|}
\hline \multirow{3}{*}{ Dimensões Pareadas } & & & & \multicolumn{6}{|c|}{ Sexo } \\
\hline & \multicolumn{3}{|c|}{ Geral } & \multicolumn{3}{|c|}{ Masculino } & \multicolumn{3}{|c|}{ Feminino } \\
\hline & $\mathrm{N}$ & $\mathrm{W}$ & $\mathrm{p}$ & $\mathrm{n}$ & W & $\mathrm{p}$ & $\mathrm{n}$ & W & $\mathrm{p}$ \\
\hline Controle de Estresse - Saúde & 50 & 106,5 & $<0,001$ & 23 & 20,0 & $<0,001$ & 27 & 37,0 & $<0,001$ \\
\hline $\begin{array}{l}\text { Controle de Estresse - } \\
\text { Sociabilidade }\end{array}$ & 50 & 448,0 & 0,314 & 23 & 154,5 & 0,371 & 27 & 75,5 & 0,034 \\
\hline $\begin{array}{l}\text { Controle de Estresse - } \\
\text { Competitividade }\end{array}$ & 50 & 1100,0 & $<0,001$ & 23 & 225,0 & $<0,001$ & 27 & 347,0 & $<0,001$ \\
\hline Controle de Estresse - Estética & 50 & 724,5 & 0,007 & 23 & 175,0 & 0,009 & 27 & 196,0 & 0,193 \\
\hline Controle de Estresse - Prazer & 50 & 272,5 & $<0,001$ & 23 & 63,0 & 0,023 & 27 & 78,0 & 0,008 \\
\hline Saúde - Sociabilidade & 50 & 699,0 & $<0,001$ & 23 & 190,0 & 0,002 & 27 & 167,0 & 0,075 \\
\hline Saúde - Competitividade & 50 & 1159,5 & $<0,001$ & 23 & 251,5 & $<0,001$ & 27 & 344,0 & $<0,001$ \\
\hline Saúde - Estética & 50 & 1166,0 & $<0,001$ & 23 & 267,5 & $<0,001$ & 27 & 328,0 & $<0,001$ \\
\hline Saúde - Prazer & 50 & 518,5 & 0,032 & 23 & 139,0 & 0,079 & 27 & 129,5 & 0,170 \\
\hline Sociabilidade - Competitividade & 50 & 1142,0 & $<0,001$ & 23 & 237,5 & $<0,001$ & 27 & 347,5 & $<0,001$ \\
\hline Sociabilidade - Estética & 50 & 862,0 & $<0,001$ & 23 & 161,5 & 0,262 & 27 & 274,5 & $<0,001$ \\
\hline Sociabilidade - Prazer & 50 & 267,5 & 0,021 & 23 & 36,0 & 0,018 & 27 & 113,5 & 0,464 \\
\hline Competitividade - Estética & 50 & 86,5 & $<0,001$ & 23 & 18,0 & $<0,001$ & 27 & 31,5 & $<0,001$ \\
\hline Competitividade - Prazer & 50 & 9,5 & $<0,001$ & 23 & 3,0 & $<0,001$ & 27 & 2,0 & $<0,001$ \\
\hline Estética - Prazer & 50 & 50,0 & $<0,001$ & 23 & 19,5 & $<0,001$ & 27 & 6,0 & $<0,001$ \\
\hline
\end{tabular}

Nota: N: Número amostral; W: Wilcoxon signed-rank test; $\mathrm{p}$ : nível de significância $(\mathrm{p} \leq 0,05)$.

Fonte: Os autores, 2019.

Os valores médios das dimensões do estado de humor estão representados no Gráfico 1. Observou-se que os participantes apresentaram um perfil iceberg após uma sessão de corrida de rua. Nota-se no Gráfico 1 que, para a dimensão Fadiga, os homens apresentaram maiores escores no momento da coleta (após uma sessão de corrida de rua) quando comparados às mulheres $(\mathrm{p}=0,039)$. Para as demais dimensões, o comportamento apresentou similaridade em ambos os grupos, não apresentando diferença significativa ( $p>0,05)$. 
Gráfico 1 - Valores médios das dimensões do estado de humor

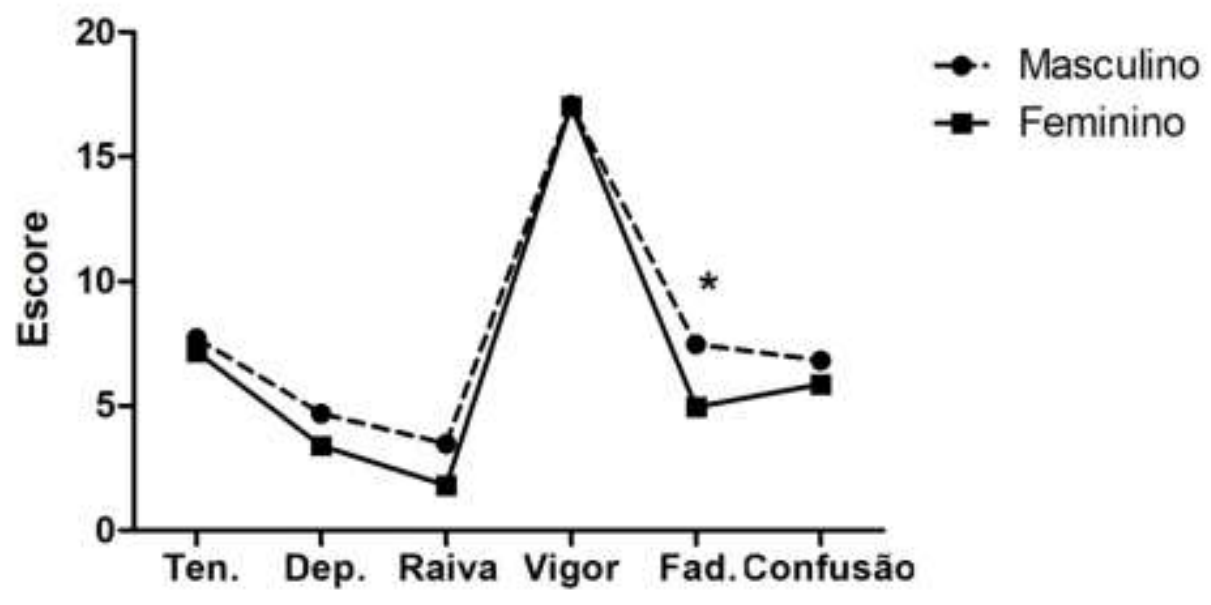

Fonte: Os autores, 2019.

\section{Discussão}

O objetivo do presente estudo foi verificar quais são as dimensões que mais motivam os corredores de rua inseridos em grupos de corrida e analisar o perfil de humor desses indivíduos. Devido aos participantes dessa pesquisa estarem inseridos em grupos de corrida, esperava-se que a dimensão Sociabilidade apresentasse escores mais altos em comparação com Controle de Estresse, Competitividade e Estética, porém isso só foi observado nas comparações do grupo das mulheres.

Conforme verificado nas comparações múltiplas realizadas para os sexos masculino e feminino entre as dimensões de motivação para a prática de corrida, a Saúde e o Prazer foram as dimensões motivacionais que mais motivaram os indivíduos praticantes de corrida de rua inseridos em grupos de corrida. O conteúdo medido pelo fator Saúde reflete os possíveis benefícios decorrentes das atividades físicas à saúde, enquanto o fator Prazer mede a experiência que os sujeitos experimentam quando atingem seus objetivos e ideais, entendendo a atividade física como uma fonte de satisfação, bem estar e auto realização, além de ser comumente apontada como a responsável pela manutenção da prática da atividade física ${ }^{24}$.

Estudos que também utilizaram o IMPRAF-54 realizados com praticantes de taekwondo $^{27}$ e praticantes regulares de atividades em academia ${ }^{28}$ encontraram resultados semelhantes, tendo a Saúde e o Prazer como as dimensões que mais motivam para a prática regular de suas respectivas atividades físicas. Em seu estudo com corredores de rua, Balbinotti et al. ${ }^{15}$ também encontraram a Saúde, o Prazer e o Controle de Estresse como as dimensões que melhor representaram os motivos de adesão e permanência à corrida de rua, e concluíram que 
as dimensões que mais estimulavam os indivíduos a praticarem regularmente o esporte não dependem do tempo de prática.

As motivações se modificam quando atletas fundistas de alto rendimento são avaliados $^{29}$. Truccollo et al. ${ }^{8}$ mostrou que razões mais importantes para as mulheres aderirem a um programa com assessoria técnica divergem das razões indicadas pelos homens, porém, aspectos como melhora do condicionamento físico e saúde, apreciar estar ao ar livre, e aumento da autoestima são comuns para ambos os sexos. Em seu estudo com corredores de rua inseridos em grupos de corrida de rua, Silva et al. ${ }^{9}$ mostrou que a saúde, prazer, estética, controle do estresse, superação de limites, e sociabilidade são os motivos e objetivos que as pessoas procuram e encontram com a prática da corrida.

A adoção e manutenção de um comportamento ativo podem levar a uma maior percepção de prazer e satisfação por parte do praticante, podendo gerar, consequentemente, uma resposta positiva no domínio psicológico neste. Repetidas experiências positivas de humor podem sustentar a motivação para prática do exercício físico ${ }^{14}$, e alguns exercícios como a corrida, são associadas ao aumento positivo das dimensões positivas do humor ${ }^{25}$. No presente estudo, nota-se que, para ambos os grupos, homens e mulheres, o perfil de humor apresentouse positivo (perfil Iceberg) ao final de uma prova de rua, sendo o grupo masculino com maiores escores para fadiga quando comparado as mulheres. Esses resultados concordam com os achados de Berger et al. $^{22}$, os quais demonstraram que corredores de rua apresentaram significativos benefícios agudos de humor a curto prazo tanto para mulheres quanto para homens, podendo ser observada melhora do humor em todas as dimensões, com exceção da Fadiga.

Um conceito clássico na Psicologia do Esporte é o "Perfil Iceberg"30", no qual os praticantes de alguma modalidade de atividade física tendem a apresentar valores superiores na dimensão positiva do humor - o Vigor - e resultados mais baixos nas dimensões negativas; Tensão, Depressão, Hostilidade, Fadiga e Confusão, quando comparados à população sedentária. Assim como neste estudo, Vieira et al. ${ }^{21}$ também mostraram que atletas corredores fundistas tendem apresentar um perfil iceberg, quando avaliados antes das sessões de treinamento.

Apesar do presente estudo auxiliar na compreensão do perfil de corredores de rua, algumas limitações devem ser mencionadas, como a quantidade de participantes e a avaliação do estado de humor em apenas em um período. Sugere-se que novos estudos, com um número maior de participantes sejam realizados a fim de verificar se existem alterações no estado de humor antes e depois de uma sessão de corrida de rua. Além disso, uma vez que este estudo 
tenha avaliado apenas corredores inseridos em grupos de corrida, seria interessante que novos estudos verificassem se corredores recreacionais independentes apresentam as mesmas características nas variáveis psicológicas de motivação e estado de humor.

\section{Conclusão}

Os resultados obtidos nesta pesquisa indicaram que as dimensões motivacionais que mais motivaram os indivíduos praticantes de corrida de rua inseridos em grupos de corrida foram a Saúde e o Prazer. Este estudo mostrou também que os indivíduos que praticam a corrida de rua tendem a apresentar uma resposta aguda positiva no perfil de humor. Pode-se concluir que a prática de corrida de rua proporciona importantes benefícios psicológicos relacionados à motivação e humor de seus praticantes.

\section{Agradecimentos}

Os autores agradecem a participação de todos os corredores de rua, grupos de corrida, equipes e treinadores envolvidos neste estudo. Este trabalho foi apoiado pela fundação CAPES, através de bolsa de fomento.

\section{Referências}

1. American College of Sports Medicine/Diretrizes do ACSM para os testes de esforço e sua prescrição; tradução Dilza Balteiro Pereira de Campos. - 9. ed. - Rio de Janeiro: Guanabara; 2014

2. Conn VS, Hafdahl AR, Brown LM. Meta-analysis of quality-of-life outcomes from physical activity interventions. Nurs Res. 2009;58(3):175-183.

3. Lima DF, Lima LA, Silva MP. Tendências temporais dos tipos principais de exercício físico e esporte praticados no lazer na cidade de Curitiba, Brasil: 2006-2014. R. bras. Ci. e Mov. 2017;25(3):98-105.

4. Bigliassi M, León-Domínguez U, Buzzachera CF, Barreto-Silva V, Altimari LR. How does music aid 5 km of running? Journal of Strength and Conditioning Research. 2015;29(2):305314.

5. Kemi O, Wisløff U. High-intensity aerobic exercise training improves the heart in health and disease. J Cardiopulm Rehabil Prev. 2010;30:2-11.

6. Salgado JVV, Chacon-Mikahil MPT. Corrida de rua: análise do crescimento do número de provas e de praticantes. Revista Conexões. 2006;4(1):100- 109. 
7. Dallari MM. Corrida de rua: um fenômeno sociocultural contemporâneo. São Paulo. Tese [Doutorado em Educação] - Faculdade de Educação, Universidade de São Paulo; 2009.

8. Truccolo, AB, Maduro PA, Feijó EA. Fatores motivacionais de adesão a grupos de corrida. Motriz: Revista de Educação Física. 2008;14(2):108-114.

9. Silva MS, Sousa MS. O papel das assessorias esportivas no crescimento das corridas de rua no Brasil. Fiep Bulletin. 2013;83.

10. Cushman MD, Babu A, Marshall B, Rho M. The motivational influence of milestone times on 10-km running performance. Int J Perform Anal Sport. 2016;16(2):602-611.

11. Chalabaev A, Radel R, Mahmoud IB, Massiera B, Deroche T, d'Arripe-Longueville F. Is motivation for marathon a protective factor or a risk factor of injury? Scand J Med Sci Sports. 2017;27:2040-2047.

12. Knechtle B. Ultramarathon Runners: Nature or Nurture? International Journal of Sports Physiology and Performance. 2012;7:310-312.

13. McCutcheon LE, Yoakum ME. Personality attributes of ultramarathoners. Journal of Personality Assessment. 1983;47(2):178-180.

14. Guérin E, Fortier MS. Situational motivation and perceived intensity: their interaction in predicting changes in positive affect from physical activity. Journal of Obesity. 2012.

15. Balbinotti MAA, Gonçalves GHT, Klering RT, Wiethaeuper D, Balbinotti CAA. Perfis motivacionais de corredores de rua com diferentes tempos de prática. Rev Bras Ciênc Esporte. 2015;37(1):65-73.

16. Buckworth J, Lee RE, Regan G, Schneider LK, Diclemente CC. Decomposing intrinsic and extrinsic motivation for exercise: application to stages of motivational readiness.

Psychology of Sport and Exercise. 2007; 8:441-461.

17. Balbinotti MAA. Inventário De Motivação À Prática Regular De Atividade Física E/Ou Esporte. Montréal, QC: Service d'Interventionet de Recherche en Orientation et Psychologie. 2004.

18. Ryan RM, Deci EL. Intrinsic and Extrinsic Motivations: Classic Definitions and New Directions. Contemporary Educational Psychology. 2000a;25(1):54-67.

19. Ryan RM, Deci EL. Self-determination theory and the facilitation of intrinsic motivation, social development, and well-being. American Psychologist. 2000b;55(1):68-78.

20. Werneck FZ, Navarro CA. Nível de atividade física e estado de humor em adolescentes. Psic.: Teor. e Pesq. 2011;27(2):189-193.

21. Vieira LF, Oliveira JS, Gaion PA, Oliveira HG, Rocha PGM, Vieira JLL. Estado de humor e periodização de treinamento: um estudo com atletas fundistas de alto rendimento. R. da Educação Física/UEM Maringá. 2010;21(4):585-591. 
22. Berger BG, Owen DR, Motl RW, Parks, L. Relationship between expectancy of psychological benefits and mood alteration in joggers. International Journal of Sport Psychology. 1998;29(1):1-16.

23. Berger BG, Motl RW, Butki BD, Martin DT, Wilkinson JG, Owen DR. Mood and cycling performance in response to three weeks of high-intensity, short-duration overtraining, and a two-week taper. The Sport Psychologist. 1999;13:444-457.

24. Barbosa MLL. Propriedades métricas do inventário de motivação à prática regular de atividade física (IMPRAF-126). Porto Alegre. Dissertação [Mestrado em Ciências do Movimento Humano] - Universidade Federal do Rio Grande do Sul; 2006.

25. Berger BG, Motl RW. Exercise and Mood: A Selective Review and Synthesis of Research Employing the Profile of Mood States. Journal of Applied Sport Psychology. 2000;12:69-92.

26. Viana MF, Almeida PL, Santos RC. Adaptação portuguesa da versão reduzida do perfil de estados de humor - POMS. Analise Psicológica. 2001;1:77-92.

27. Sampedro LBR, Rocha JM, Klering RT, Saldanha RP, Balbinotti MAA, Balbinotti CAA. Motivação à prática regular de atividades físicas: um estudo com praticantes de taekwondo. Pensar a Prática. 2014;17(1):1-294.

28. Passos JR, Passos JR, Chaves GFT, Portes LA, Oliveira NC. Motivação para a prática de atividades físicas em academias. LifeStyle Journal. 2016;3(2):59-67.

29. Zaar A, Balbinotti MAA. Um estudo descritivo das dimensões motivacionais dos atletas corredores de 5.000 e 10.000 metros do Troféu Brasil de atletismo. EF Deportes, Revista Digital. 2011;15(152). Disponível em: <http://www.efdeportes.com>. Acesso em: $01 \mathrm{de}$ Março de 2018.

30. Morgan WP. Test of champions: the iceberg profile. Psychology Today. 1980;14(4):92108. 